Journal of Molecular Structure, 22 (1974) 61-67

(C) Elsevier Scientific Publishing Company, Amsterdam - Printed in The Netherlands

\title{
THE MICROWAVE SPECTRUM OF MONOCHLOROACETIC ACID
}

B. P. VAN EIJCK, A. A. J. MAAGDENBERG AND J. WANROOY

Laboratorium voor Kristalchemie der Rijksuniversiteit, Catharijnesingel 51, Utrecht (The. Netherlands)

(Received 10 September 1973)

\section{ABSTRACT}

The microwave spectrum, rotational constants and centrifugal distortion parameters for $\mathrm{CH}_{2}{ }^{35} \mathrm{ClCOOH}$ are reported. The nuclear quadrupole coupling constants are: $\chi_{\mathrm{as}}=-47.5 \pm 1.4 \mathrm{MHz}, \chi_{\mathrm{bb}}=10.4 \pm 0.7 \mathrm{MHz}, \chi_{\mathrm{cc}}=37.1 \pm 1.2$ $\mathrm{MHz}$, and the dipole moment components: $\mu_{\mathrm{a}}=1.658 \pm 0.008 \mathrm{D}, \mu_{\mathrm{b}}=2.112$ $\pm 0.006 \mathrm{D}(\mu=2.685 \pm 0.006 \mathrm{D})$. From a-substitution coordinates of the $\mathrm{Cl}$ atom and the carboxyl $\mathrm{H}$ atom the chain of atoms $\mathrm{Cl}-\mathrm{C}-\mathrm{C}-\mathrm{O}-\mathrm{H}$ is shown to be planar and zig-zag. An estimate of the torsional frequency is given.

\section{INTRODUCTION}

In our investigation of the molecular structure of fluoroacetic acid [1] it was found that free molecules of this substance may occur in at least two conformations. A study of chloroacetic acid was undertaken in order to investigate the degree of structural similarity between both compounds. In this paper we describe the microwave spectrum; results of electron diffraction work will be reported by Derissen and Bijen [2].

\section{EXPERIMENTAL}

Chloroacetic acid was obtained commercially and purified by low pressure sublimation. Deuterium was introduced into the carboxyl group by reaction of $\mathrm{D}_{2} \mathrm{O}$ with chloroacetylchloride. This compound was prepared from chloroacetic acid and $\mathrm{PCl}_{3}$, as described by Vogel [3] for acetylchloride.

The microwave spectrometer was a conventional $10 \mathrm{kHz}$ Stark-modulated 
instrument. The spectra $(20-39 \mathrm{GHz})$ were recorded at room temperature at a pressure of about $0.03 \mathrm{~mm} \mathrm{Hg}$. The accuracy of the frequency measurements was about $0.2 \mathrm{MHz}$.

\section{ANALYSIS}

The spectrum was found to be very rich in lines. The dominating ones are Q-type transitions $J_{2, J-2}-J_{3, J-3}$ with $J$ ranging from 10 to 22 (Table 1). They were identified with aid of a numerical method described earlier [4]. After that, R-type lines were found easily.

\section{TABLE 1}

ABSOR PTION FREQUENCIES (average values of quadrupole componentS) FOR THE STRONGEST TRANSITIONS IN THE VIBRATIONAL GROUND STATE

\begin{tabular}{|c|c|c|c|c|c|c|}
\hline \multirow[t]{2}{*}{ Transition } & \multicolumn{2}{|c|}{$\mathrm{CH}_{2}{ }^{35} \mathrm{ClCOOH}$} & \multicolumn{2}{|c|}{$\mathrm{CH}_{2}{ }^{37} \mathrm{ClCOOH}$} & \multicolumn{2}{|c|}{$\mathrm{CH}_{2}{ }^{35} \mathrm{ClCOOD}$} \\
\hline & $\boldsymbol{v}_{\text {obs }}$ & $\Delta v$ & vots & $\Delta v$ & $y_{\text {oths }}$ & $\Delta v$ \\
\hline $\begin{array}{l}10_{2,8}-10_{3,7} \\
11_{2,9}-11_{3,8}\end{array}$ & $\begin{array}{l}37958.4 \\
36778.0\end{array}$ & $\begin{array}{r}-0.14 \\
+0.18\end{array}$ & 37397.0 & -0.02 & & \\
\hline $12, .10-123.9$ & 35550.1 & +0.15 & 36202.7 & -0.01 & 36435.7 & -0.06 \\
\hline $132,11-13,10$ & 34361.0 & -0.22 & & & 35252.3 & +0.00 \\
\hline $14_{2.12}-14_{3.11}$ & 33303.9 & -0.09 & 33920.8 & -0.00 & 34148.1 & +0.15 \\
\hline $15_{2.13}-15_{3.12}$ & 32473.6 & -0.19 & 33006.3 & +0.03 & 33209.1 & -0.14 \\
\hline $16_{2,14}-16_{3,13}$ & 31957.0 & -0.04 & 32356.2 & -0.03 & 32517.0 & +0.07 \\
\hline $17_{2.15}-17_{3.14}$ & 31830.6 & +0.08 & 32045.7 & +0.05 & 32147.5 & -0.01 \\
\hline $18_{2,16}-183,15$ & 32157.5 & $\div 0.19$ & 32139.2 & +0.01 & 32164.6 & -0.06 \\
\hline $19_{2,17}-19_{3,16}$ & 32988.0 & -0.07 & 32688.7 & +0.02 & 32620.9 & +0.15 \\
\hline $20_{2.18}-20_{3.17}$ & 34358.3 & +0.11 & 33734.9 & -0.10 & 33558.5 & +0.01 \\
\hline $21_{2.19}-21_{3.18}$ & 36292.4 & -0.12 & 35306.2 & -0.06 & 35007.2 & -0.02 \\
\hline $222.20-223.19$ & 38796.4 & -0.14 & 37418.0 & +0.09 & 36984.9 & -0.03 \\
\hline
\end{tabular}

$\Delta \nu \equiv v_{\text {calc }}-v_{\text {obs }}$.

As for fluoroacetic acid, lines belonging to a number of excited torsional states were observed. For states with torsional quantum number $v$ greater than 4 the R-type transitions were too weak to be identified, so that no complete set of rotational constants could be obtained. The same applies to the first excited state of a skeletal bending vibration.

The spectrum of the ${ }^{37} \mathrm{Cl}$ species was identified too. Here, as well as in the case of $\mathrm{CH}_{2}{ }^{35} \mathrm{ClCOOD}$, only lines belonging to the ground state were analyzed.

A selection of strong ground state transitions, which might be useful for "fingerprinting" the spectrum, is given in Table 1. For these transitions the quadrupole splitting is small, no component being further than $1 \mathrm{MHz}$ away from the centre frequency. A complete list of measured frequencies is available from the 


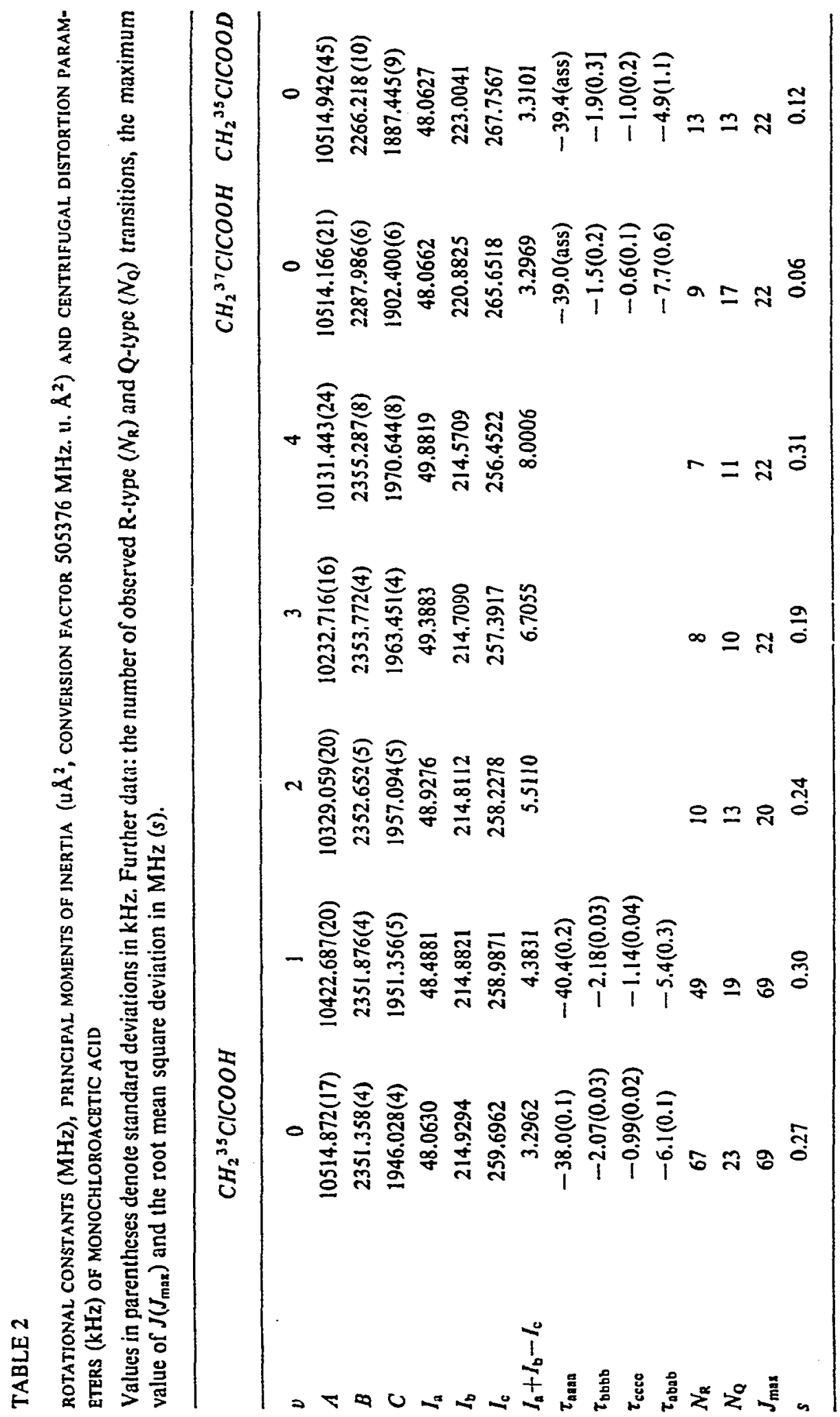


authors on request. The molecular parameters that were calculated from the measured centre frequencies are given in Table 2 . In the least squares procedure centrifugal distortion parameters for most vibrational states were kept fixed at values taken from lower states.

Many lines showed quadrupole splitting due to the chlorine nucleus. For all well-split transitions we could find, the splitting was essentially symmetric with respect to the hypothetical unsplit line. In fact, in the majority of cases there only was a symmetrical doublet with one component due to $F=J \pm \frac{3}{2}$ and the other to $F=J \pm \frac{1}{2}$. From 35 of these doublet splittings in $\mathrm{CH}_{2}{ }^{35} \mathrm{ClCOOH}$ (Table 3) we

\section{TABLE 3}

OBSERVED AND CALCUlated QuAdRufole SPLitTings (MHz) in $\mathrm{CH}_{2}{ }^{35} \mathrm{ClCOOH}$

Root mean square deviation $0.17 \mathrm{MHz} . \Delta v \equiv v_{F=J \pm}-v_{F=J \pm 1}$.

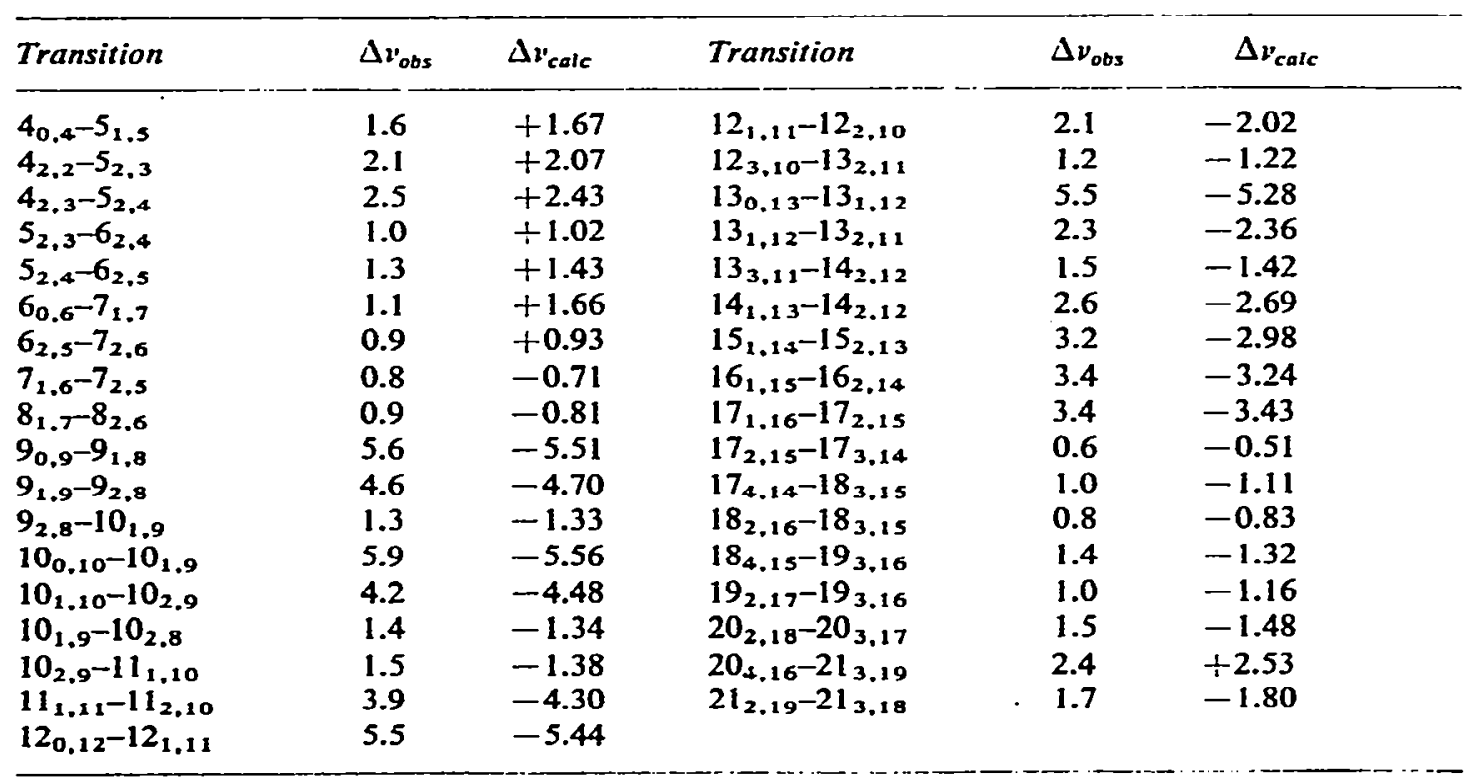

calculated the quadrupole coupling constants by the least squares method: $\chi_{a \mathrm{a}}=$ $-47.5 \pm 1.4 \mathrm{MHz}, \chi_{\mathrm{bb}}=10.4 \pm 0.7 \mathrm{MHz}, \chi_{\mathrm{cc}}=37.1 \pm 1.2 \mathrm{MHz}$. For the other isotopic species not enougl: splittings were observed to derive reliable $\chi$-values.

A sign reversal of all three quadrupole coupling constants would produce the same doublet splittings. The signs given above are derived by assuming that the principal quadrupole axis $\alpha$ coincides with the $\mathrm{C}-\mathrm{Cl}$ bond and that $\chi_{\alpha}$ is negative, as usual [5]. Taking the $(\alpha, a)$ angle from a molecular model [2] to be $29^{\circ}$, we calculate $\chi_{\alpha}=-73.3 \pm 2.3 \mathrm{MHz}$ and $\eta \equiv\left(\chi_{\beta}-\chi_{c}\right) / \chi_{\alpha}=0.012 \pm 0.013$. Within our experimental error the charge distribution is, therefore, cylindrically symmetric around the $\mathrm{C}-\mathrm{Cl}$ bond. A literature value [6] for $\left|\chi_{\alpha}\right|$ in the solid state is $\mathbf{7 3 . 1 5}$ MHz. 


\section{Dipole moment}

The Stark shifts of a number of transitions were measured in the usual way, the frequency shift of the $J=1 \rightarrow 2$ transition in OCS [7] being used to calibrate the absorption cell. Fourth-order terms in the field strength were taken into account:

$$
\begin{aligned}
& \Delta v / E^{2}=c^{(2)}+c^{(4)} E^{2} \\
& c^{(2)}=k_{\mathrm{a}} \mu_{\mathrm{a}}{ }^{2}+k_{\mathrm{b}} \mu_{\mathrm{b}}{ }^{2}
\end{aligned}
$$

From the observed values of $\Delta v / E^{2}$ the quantity $c^{(4)} E^{2}\left(c^{(4)}\right.$ being calculated by the formula given in ref. 1) was subtracted in order to find $c^{(2)}$. This correction ranged from $1 \%$ to $9 \%$ of $c^{(2)}$. From six values of $c^{(2)}$ (Table 4) the dipole moment components were calculated by the least squares method: $\mu_{a}=1.658 \pm 0.008$ $\mathrm{D}, \mu_{\mathrm{b}}=2.112 \pm 0.006 \mathrm{D} ;|\mu|=2.685 \pm 0.006 \mathrm{D}$.

TABLE 4

COMPARISON OF CALCULATED AND OBSERVED STARK COEFFICIENTS $\mathrm{c}^{(2)}$ IN $\mathrm{CH}_{2}{ }^{35} \mathrm{CICOOH}$ $\Delta y$ in $\mathrm{MHz}, E$ in Volt $\mathrm{cm}^{-1}, \mu$ in Debye; conversion factor $\mu E=0.50344 \mathrm{MHz} \mathrm{cm} \mathrm{D}^{-1}$ Volt $^{-1}$; $A \equiv 10^{8}\left|c_{\mathrm{obs}}^{(2)}-c_{\mathrm{calc}}^{(2)}\right|$.

\begin{tabular}{llllcccc}
\hline Transition & $M$ & $10^{8} k_{a}$ & $10^{8} k_{b}$ & $10^{8} c_{o b s}^{(2)}$ & $10^{8} c_{c a l c}^{(2)}$ & 1 & $10^{16} c_{c a l c}^{(1)}$ \\
\hline $5_{24-6}-6_{25}$ & 1 & +159.82 & +89.29 & $+853 \pm 8$ & +838 & 15 & -2520 \\
$6_{06}-7_{07}$ & 1 & -8.794 & -8.096 & $-60.32 \pm 0.11$ & -60.29 & 0.03 & -1.90 \\
$7_{07-808}$ & 3 & -2.422 & +30.88 & $+132.0 \pm 0.6$ & +131.1 & 0.9 & -5.98 \\
& 1 & -7.156 & -5.802 & $-45.2 \pm 0.2$ & -45.6 & 0.4 & -2.90 \\
$8_{08}-9_{09}$ & 3 & -5.472 & +16.910 & $+59.9 \pm 0.4$ & +60.4 & 0.5 & -7.37 \\
& 1 & -8.370 & -4.409 & $-43.0 \pm 0.3$ & -42.7 & 0.3 & -16.5 \\
\hline
\end{tabular}

In the six transitions studied no effects of quadrupole splitting were observed. Yet a comparison of calculated quadrupole splittings and Stark shifts showed that the "strong-field case" was by no means reached for all measurements, and that especially for the $M_{J}= \pm 1$ levels complications should be expected. The fact that no anomalies were observed must be ascribed to a fortuitous cancelling of quadrupole effects when a transition frequency is calculated as the difference between two energy levels disturbed in about the same way.

\section{Torsional motion}

The torsional frequency $\sigma_{\mathrm{\imath}}$ might be found from relative intensity measurements on rotational transitions in the various torsional states, but because of the density of the spectrum and the complications due to quadrupole splitting we did not attempt to do this. Instead, an estimate was made from the relation $[1,8]$ between the $I_{\mathrm{a}}+I_{\mathrm{b}}-I_{\mathrm{c}}$ values and the torsional frequency, giving $\sigma_{\mathrm{t}}=62 \mathrm{~cm}^{-1}$. 
A much larger value [9] of $243 \mathrm{~cm}^{-1}$ used by Johansen [10] must be in error, since we observed lines due to excited torsional states up to $v=6$. In fact, we believe Johansen's force field to have more defects, since centrifugal distortion parameters calculated from this field were 2 to 10 times greater than our experimental values.

\section{Molecular structure}

From the values of $I_{\mathrm{a}}+I_{\mathrm{b}}-I_{\mathrm{c}}$ (Table 2), and the fact that the rotational constants change smoothly with the torsional quantum number $v$, we deduce that the molecule has a plane of symmetry.

In principle the substitution coordinates of the $\mathrm{Cl}$ and $\mathrm{H}$ atoms could be obtained from the data available. However, both atoms turn out to have very small $b$-coordinates; the $b_{H}$, in fact, would even be found imaginary. Only the a-coordinates are reliable; they are: $\mathrm{a}_{\mathrm{Cl}}=1.7448 \pm 0.0002 \AA$ and $\mathrm{a}_{\mathrm{H}}=-2.8478 \pm$ $0.0002 \AA$. From the $\mathrm{H} \cdots \mathrm{Cl}$ distance of $4.5926 \AA$ the molecular conformation is immediately obvious:<smiles>O=C(O)Cl</smiles>

The molecular structure is being investigated by electron diffraction by Derissen and Bijen. The inertial moments and substitution coordinates calculated from their preliminary bond distances and bond angles agree fairly with our results. A full account will be published [2]. The electron diffraction data indicate the presence of at least one more conformation, formed by rotating the carboxyl group $130^{\circ}$ about the $\mathrm{C}-\mathrm{C}$ axis. We have searched carefully for absorption lines belonging to this molecular species, but failed to make an identification. An approximate prediction of the spectrum of this rotamer showed that only for high $J$-values lines may be expected that compete in intensity with the many weak lines of the first conformation and its vibrationally excited states. Therefore, we doubt that we shall be able to make an assignment for this second conformation.

\section{ACKNOWLEDGEMENTS}

Many thanks are due to Dr. H. A. Dijkerman and Mr. Th. Ram for their kind assistance in the microwave experiments. We are also grateful to Dr. J. L. Derissen and to Dr. G. O. Sørensen for helpful discussions. 


\section{REFERENCES}

1 B. P. van Eijck, G. van der Plaats and P. H. van Roon, J. Mol. Structure, 11 (1972) 67.

2 J. L. Derissen and J. M. J. M. Bijen, J. Mol. Structure, to be published.

3 A. I. Vogel, A Textbook of Practical Organic Chemistry, Longmans, London 3rd ed., 1956, p. 367.

4 B. P. van Eijck, J. Mol. Spectrosc., 38 (1971) 149.

5 E. A. C. Lucken, Nuclear Quadrupole Coupling Constants, Academic Press, London and New York, 1969, p. 168.

6 H. C. Allen Jr., J. Amer. Chem. Soc., 74 (1952) 6074.

7 J. S. Münther, J. Chem. Phys., 48 (1968) 4544.

8 J. H. Høg, L. Nygaard and G. O. Sørensen, J. Mol. Structure, 7 (1970) 111.

9 J. R. Barceló, M. Pilar Jorge and C. Otero, J. Chem. Phys., 28 (1958) 1230.

10 H. Johansen, Z. Phys. Chem., 227 (1964) 305. 